\title{
Mycobacterium chelonae Is an Ubiquitous Atypical Mycobacterium
}

\author{
Miguel Pinto-Gouveia Ana Gameiro Leonor Ramos \\ José Carlos Cardoso Maria Manuel Brites Óscar Tellechea \\ Américo Figueiredo \\ Dermatology Department, Coimbra University Hospital Centre, Coimbra, Portugal
}

\section{Key Words}

Immunosuppression · Mycobacterium chelonae $\cdot$ Atypical mycobacteria $\cdot$ Corticosteroid therapy

\begin{abstract}
The type of cutaneous infection varies mainly according to the patient's immune status, and the disseminated form is mostly found in the context of immunosuppression. We report the case of a 62-year-old male who was under long-term systemic corticosteroid therapy and presented with a 7-month history of multiple painless cutaneous lesions at various stages of development: papules, nodules, pustules and hemorrhagic crusts, as well as small erosions and ulcers distributed over the limbs and scalp. Cutaneous biopsy showed a suppurative granulomatous infiltrate with abscess formation. Fite stain revealed numerous extracellular bacilli, suggesting mycobacterial infection, particularly by atypical mycobacteria. Culture of a skin sample revealed Mycobacterium chelonae. The patient started multidrug therapy and showed clinical improvement despite of resistance to one of the antibiotics. This striking presentation underlines the role of immunosuppression with corticotherapy as a major risk factor for these infections. Multidrug therapy is advised and antibiogram is essential in directing treatment.

\section{Introduction}

Mycobacterium chelonae is a rare atypical mycobacterium. The type of cutaneous infection varies mainly according to the patient's immune status, and the disseminated form is usually found in the context of immune depression. 


\section{Case Reports in Dermatology}

Case Rep Dermatol 2015;7:207-211

\begin{tabular}{l|l}
\hline DOI: $10.1159 / 000438898$ & (c) 2015 The Author(s). Published by S. Karger AG, Basel \\
\end{tabular}
www.karger.com/cde

Pinto-Gouveia et al.: Mycobacterium chelonae Is an Ubiquitous Atypica Mycobacterium

\section{Case Presentation}

We report the case of a 62-year-old male, who was under systemic corticosteroid therapy (prednisolone $60 \mathrm{mg} /$ day) for about 5 years, for unconfirmed dermatomyositis, initially hospitalized for strangulated inguinoscrotal hernia complicated with Fournier gangrene.

During hospitalization, dermatology observation was requested for a 7-month history of multiple painless and nonpruritic cutaneous lesions at various stages of development: erythematous papules and nodules, pustular lesions as well as hemorrhagic crusts, small erosions and ulcers that resolved with depressed cicatricial lesions (fig. 1), distributed over the upper and lower limbs, but also on the scalp and face, sparing the trunk (fig. 2).

He had no systemic symptoms, and besides the surgical wound in the right inguinal region, there were no other abnormalities in the physical examination.

Cutaneous biopsy of a pustule showed a suppurative granulomatous infiltrate with abscess formation. Fite stain revealed numerous extracellular bacilli, suggesting mycobacterial infection, particularly by atypical mycobacteria (fig. 3).

The blood and urine cultures for mycobacteria were negative. HIV serology was negative, blood count was normal, and chest CT scan did not show evidence of infectious or tumoral lesions. Culture of a skin sample revealed M. chelonae, thus establishing the diagnosis of disseminated M. chelonae infection in the context of immunosuppression.

While antibiogram was pending, oral clarithromycin and ciprofloxacin were started, due to the necessity of prolonged therapy and avoidance of antibiotic resistance, and intravenous amikacin was added for the first 3 weeks considering the extension of the disease.

After 45 days of therapy, the patient showed significant improvement, with fewer pustular and exudative lesions. However, the patient underwent several surgeries regarding the Fournier gangrene and died in the surgery ward due to postsurgical complications. Antibiogram later revealed susceptibility to clarithromycin and amikacin, but resistance to ciprofloxacin.

\section{Discussion}

We presented the case of an immunocompromised patient under prolonged systemic corticosteroid therapy with an extensive disseminated cutaneous $M$. chelonae infection.

$M$. chelonae is a nontuberculous, rapidly growing species of mycobacteria and is often grouped together with $M$. fortuitum and $M$. abscessus as the Fortuitum complex due to their similar metabolic and clinical features [1].

It is found in soil, dust and natural water supplies. Infection typically follows incidental environmental inoculation, but it has also been associated with surgeries [2], tattooing [3] and cosmetic procedures such as liposuction [4].

The cutaneous manifestations vary mainly according to the patient's immune status: localized infections such as cellulitis and abscesses commonly present in immunocompetent patients, whereas disseminated skin disease occurs in the background of immunosuppression, although a case in an immunocompetent host has been described [5]. In disseminated disease, limbs are predominantly involved with more than 5 lesions (pustules, nodules, abscesses or ulcers), commonly showing sporotrichoid pattern, and may develop without any trauma $[6,7]$. Our case is impressive because of the numerous lesions over limbs and scalp, with remarkable sparing of the trunk.

Other less common presentations reported in the literature include osteomyelitis, pneumonitis, lymphadenitis, corneal ulcers, endocarditis or catheter-related infections [8]. 
Pinto-Gouveia et al.: Mycobacterium chelonae Is an Ubiquitous Atypical Mycobacterium

Underlying conditions for disseminated disease include organ transplantation, chemotherapy and autoimmune disorders. Long-term steroid therapy with doubtful indication certainly played an important role in our patient's infection, and corticosteroid usage has in fact been reported in $92 \%$ of cases [9], which makes it a major risk factor for these infections [10].

In addition, there have been several of cases of M. chelonae infections associated with biologic therapies [11], and although the most common mycobacterial infections in these patients are caused by M. tuberculosis, the widespread use of these drugs may originate an increasing number of infections by opportunistic agents like atypical mycobacteria.

Most standard antimycobacterials such as ethambutol, pyrazinamide and isoniazid show little or no effect against the rapidly growing mycobacteria [12]. Clarithromycin alone was shown to be an adequate treatment; however, acquired resistance has been reported, seemingly caused by a mutation in the gene coding $23 \mathrm{~S}$ ribosome RNA [13]. Furthermore, resistance of $M$. chelonae to other antimicrobials such as erythromycin, ciprofloxacin, ofloxacin and sulfamethoxazole has been described [9]. Hence, current literature supports multidrug therapy to avoid selecting resistant strains and a prolonged therapy period in order to prevent relapse [10].

Obtaining in vitro susceptibilities is essential in directing treatment [8], but since it usually takes some time to obtain the antibiogram, suggested initial regimens include clarithromycin with the addition of imipenem, tobramycin or amikacin $[12,14]$. Many other newer antibiotics were successful in treating $M$. chelonae infections, like faropenem [13] and roxithromycin [15], and other treatment modalities should be considered in more recalcitrant or small lesions, such as surgical excision or cryotherapy [10].

We report this case because of its striking and still unusual clinical presentation, which provides a good example of the role of immunosuppression with corticosteroids as a major risk factor for nontuberculous mycobacteria infection, such as $M$. chelonae. Multidrug therapy is advised to prevent antibiotic resistance, and antibiogram should be performed to confirm susceptibility, even in the setting of early clinical improvement.

\section{Statement of Ethics}

The authors declare no ethical violations.

\section{Disclosure Statement}

The authors have no financial support and no conflicts of interests to declare.

\section{References}

1 Kullavanijaya P: Atypical mycobacterial cutaneous infection. Clinics Dermatol 1999;17:153-158; discussion 105-106.

2 Thomas M, D'Silva JA, Borole AJ, Chilgar RM: Periprosthetic atypical mycobacterial infection in breast implants: a new kid on the block! J Plast Reconstr Aesthet Surg 2013;66:e16-e19.

-3 Goldman J, Caron F, de Quatrebarbes J, Pestel-Caron M, Courville P, Dore MX, et al: Infections from tattooing. Outbreak of Mycobacterium chelonae in France. BMJ 2010;341:c5483.

4 Kim MJ, Mascola L: Mycobacterium chelonae wound infection after liposuction. Emerg Infect Dis 2010;16:1173-1175. 
Pinto-Gouveia et al.: Mycobacterium chelonae Is an Ubiquitous Atypical Mycobacterium

5 Halpern J, Biswas A, Cadwgan A, Tan BB: Disseminated cutaneous Mycobacterium chelonae infection in an immunocompetent host. Clin Exp Dermatol 2010;35:269-271.

6 Chung WK, Kim MS, Kim CH, Lee MW, Choi JH, Moon KC, et al: Cutaneous Mycobacterium chelonae infection presenting as symmetrical plaques on both shins in an immunocompetent patient. Acta Derm Venereol 2009;89:663-664.

7 Haas SR, Hodge MB, Duncan RA: Cushing's syndrome presenting as disseminated cutaneous Mycobacterium chelonae infection. Clin Infect Dis 2001;33:e51-e53.

-8 Kane CL, Vincent AL, Greene JN, Sandin RL: Disseminated cutaneous Mycobacterium chelonae infection. Cancer Control 2000;7:181-184.

-9 Wallace RJ, Jr., Brown BA, Onyi GO: Skin, soft tissue, and bone infections due to Mycobacterium chelonae chelonae: importance of prior corticosteroid therapy, frequency of disseminated infections, and resistance to oral antimicrobials other than clarithromycin. J Infect Dis 1992;166:405-412.

10 Kullavanijaya P, Rattana-Apiromyakij N, Sukonthapirom-Napattalung P, Sirimachand S, Duangdeeden I: Disseminated Mycobacterium chelonae cutaneous infection: recalcitrant to combined antibiotic therapy. J Dermatol 2003;30:485-491.

-11 Conejero R, Ara M, Lorda M, Rivera I: Mycobacterium chelonae infection in a patient being treated with adalimumab. Actas Dermosifiliogr 2012;103:69-71.

12 Iseman M: Group IV: rapid growing mycobacteria; in Yu VL, Merigan TC, Barriere SL (eds): Antimicrobial Therapy and Vaccines. Philadelphia, William \& Wilkins, 1999, pp 494-497.

$\checkmark 13$ Morihara K, Takenaka H, Morihara T, Katoh N: Cutaneous Mycobacterium chelonae infection successfully treated with faropenem. J Dermatol 2011;38:211-213.

14 Shim TN, Lew TT, Preston PW: Disseminated cutaneous Mycobacterium chelonae. Lancet Infect Dis 2012;12:254.

15 Sodemoto K, Shimada Y, Nishijima C, Inaoki M: Successful treatment of cutaneous Mycobacterium chelonae infection with roxithromycin. J Dermatol 2007;34:846-848.
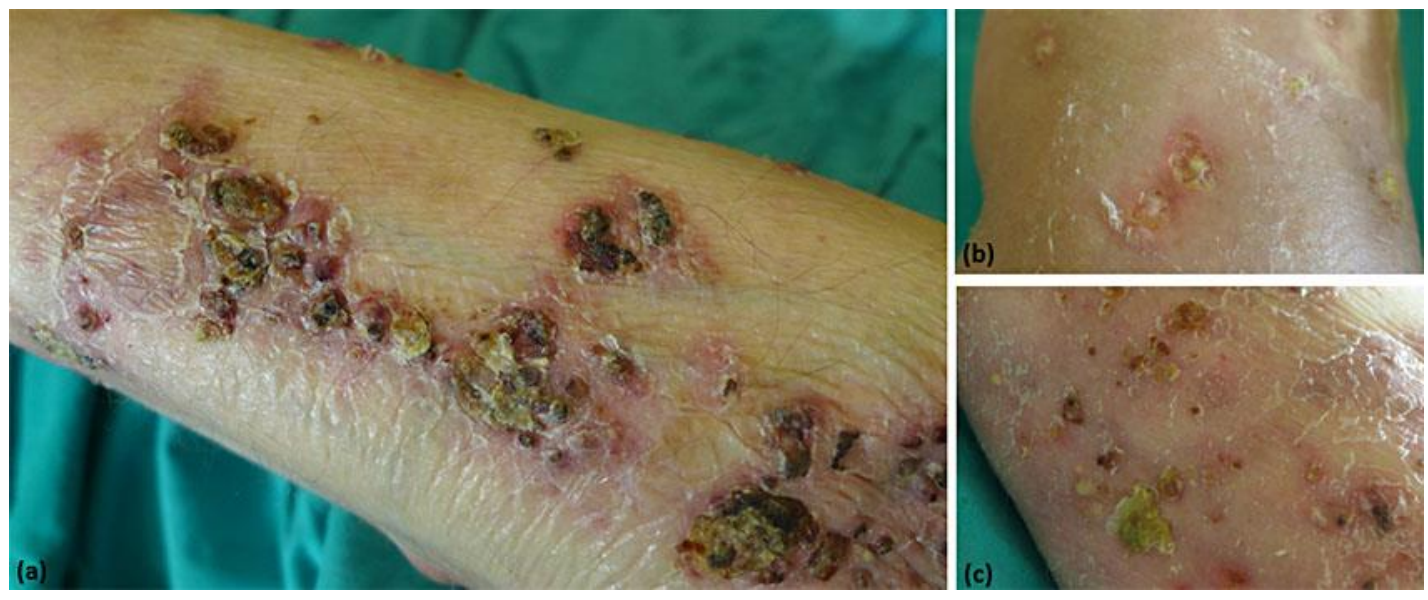

Fig. 1. Cutaneous lesions at various stages of development. a Erythematous papules and nodules, and hemorrhagic crusts. b Small erosions and ulcers. c Pustules and depressed cicatricial lesions. 


\section{Case Reports in Dermatology}
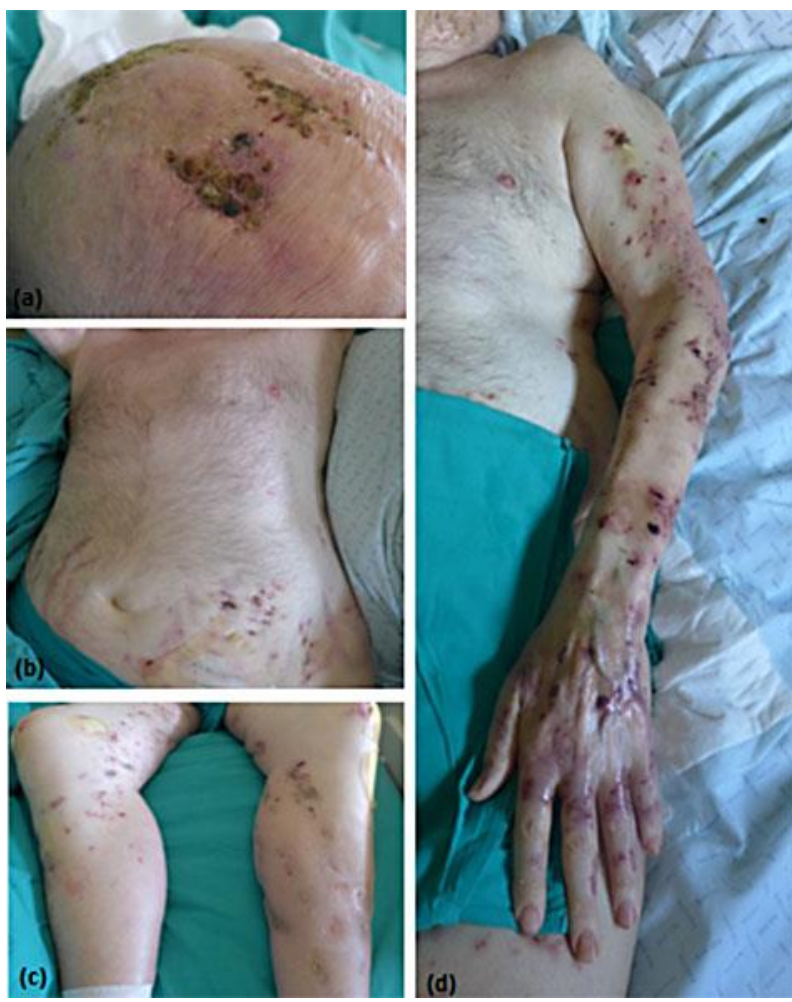

Fig. 2. Distribution of lesions: sporotrichoid pattern over the limbs (c, d) and scalp (a), with sparing of the trunk (b).
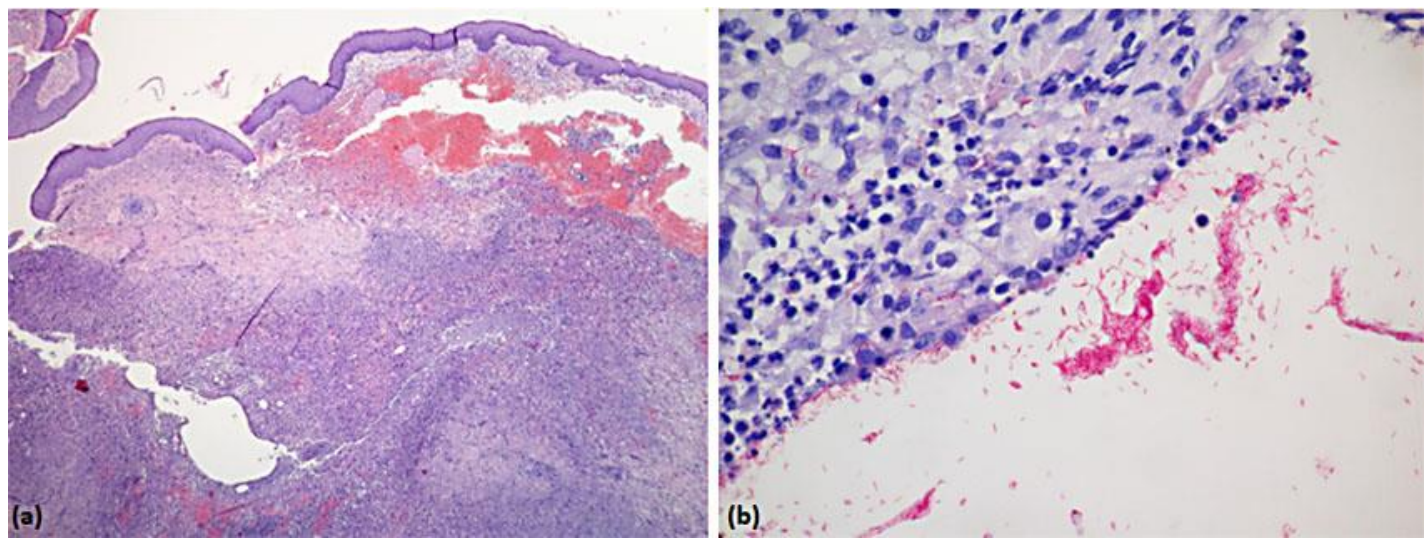

Fig. 3. Histopathology of pustular lesion. Suppurative granulomatous infiltrate with abscess formation (a). Fite stain revealed numerous extracellular bacilli, frequently forming clumps (b). 